\title{
MANAGEMENT OF CONSUMPTION ACCORDING TO THE SPECIAL ROLE OF NEED FOR SAFETY
}

\author{
Oleg Avilov $1, \mathrm{a}, *$ \\ ${ }^{1}$ Pobedy ave., 166b, fl.13, 454084 Russia \\ aavilveschun@gmail.com \\ ${ }^{*}$ Corresponding author
}

Cite as: Avilov, O. (2021). Management of consumption according to the special role of need for safety, Ekonomicko-manazerske spektrum, 15(1), 75-83.

Available at: dx.doi.org/10.26552/ems.2021.1.75-83

Received: 16 December 2020; Received in revised form: 17 March 2021; Accepted: 21 April 2021; Available online: 28 April 2021

\begin{abstract}
The article, based on the theory of functional equivalence, explains the reasons for changing individual consumption. From the point of view of the theory mentioned, any need is an imbalance caused by an excess of information, physical and chemical factors that arise during life. Satisfaction of a need is the search for a "channel" to establish equivalence. Such "channel" can represent either an increase or a decrease in number of factors used to eliminate the imbalance. The article shows that one of the most common imbalances is lack of security and proposes five "channels" to eliminate this imbalance. This imbalance is presented as something that already exist at the level of zygote and is determined by the initial level of disequilibrium which in turns determines future potential of the organism in question. This potential then has to be realized in the course of individual development. At the same time, additional imbalances generated, in particular by advertising, can lead to increased spending and risk of disease. In the process of searching for "channels" to establish equivalence, emotional stress develops. Satisfaction of needs is accompanied by a decrease in the severity of emotional stress and formation of positive emotions. In a survey of students, it was found that those who gave great importance to eliminating the lack of feeling of security by means of gaining new knowledge had lower levels of stress and more positive emotions than those who tried to eliminate their imbalances by accumulating material goods. The article shows that through coaching, individual hierarchy of needs may be changed.
\end{abstract}

Keywords: functional equivalence theory; needs; potential; stress; students

JEL Classification: E21

\section{Introduction}

Governments of all countries try to support economic development. At the same time, economic prosperity, according to most economists, involves constant increase in the volume of consumption of goods and services. The population of most countries of the world is used to such consumption. Moreover, in developed countries, any restriction on consumption is seen as an attack on individual freedom (Reith, 2004). On the other hand, voices are becoming louder saying that further unrestricted growth in consumption can be destructive for both nature and the mankind (Avilov, 2020). One of the most prominent authorities calling for reduced consumption is probably the Dalai Lama. In one of his interviews, he said: "Some desires are 
positive - for example, the desire for happiness. Some desires are very useful. However, at some point, desires may become unreasonable. As a rule, this leads to trouble. So, for example, now I sometimes visit super-markets. I really like it there, as I see so many beautiful things. When I look at all this variety of objects, I have a desire and an initial impulse like 'oh, I want this; I want that.' Then, thinking about it, I ask myself: 'Do I really need this? The answer is usually no. If we follow this first wish, our pockets will quickly empty. Reasonable desires that belong to another level and are based on primary needs - the need for food, clothing, and a roof over your head [...]"'. According to the Dalai Lama, there are two methods of achieving inner satisfaction. The first is to get everything we want and desire-money, houses, cars, a perfect partner, a perfect body. The Dalai Lama points out the disadvantages of this approach; if we lose control of our desires, sooner or later we will stumble upon something that we want but cannot get. The second, more reliable method, is not to have what we want to have, but to both want and appreciate what we have (The Dalai Lama, 2004).

Is the Dalai Lama right? Is it possible to find scientific confirmation of the correctness of his reasoning? What to buy? How much to buy? What does the pleasure of buying, of gaining something depends on? To answer these questions, we propose to use our theory of functional equivalence.

\section{Methodology}

Within the framework of methodological approaches of the theory of functional equivalence, we believe that information, physical and chemical effects on the body should be equivalent to the sum of the information, physical and chemical components of vital activity transformed in the body and isolated from the body (Avilov, 2019). Using this point of view, we can offer a different view of understanding of what a need is. In our opinion, a need is the development of an imbalance of varying degrees of severity, caused by an excess of information, physical and chemical factors formed in the process of vital activity, which cannot be removed by the body independently, without the risk of violating the structure (Mazonna and Peracchi, 2017). Satisfaction of needs is then the search for "channels" to establish equivalence, that is, an increased or decreased amount of information, physical and chemical incentives to eliminate this excess. The ultimate end of satisfying one's needs is the establishment of equivalence by converting the excess of information, physical and chemical components of vital activity into functional and structural changes in the body. Such as, for example, the elimination or synthesis of certain substances, the renewal of cellular composition, changes in the mass and volume of organs and tissues, changes in motor and behavioral activity. The process of establishing equivalence can take place in stages. At the same time, the sum of physical, chemical, and informational changes in the body after satisfaction of needs is equivalent to those before such satisfaction (Fawsy and Hamed, 2017). It is only the relationship between the physical, chemical, and informational components of the life process that changes. The formation and elimination of imbalances occurs continuously throughout life. From the point of view of our theory, the imbalance already exists at the level of zygote and is determined by the initial level of disequilibrium. The step-by-step process of forming equivalence allows one to slow down the process of approaching full equilibrium-death. The elimination of imbalances without consequences for the processes of vital activity is possible only in the volume and pace that are determined mainly by the genetic characteristics of the body. By and large, the meaning of our life is elimination of imbalances at the optimal pace for delaying the time of death (Avilov, 2019). At the same time, elimination of an imbalance of any degree of severity is always accompanied by positive emotions. 
To find out which needs, or imbalances, are most relevant to students, they were asked to rate a list of needs based on their subjective preference. The study involved one hundred and fifty-seven students of higher educational institutions of the city of Chelyabinsk aged twenty to twenty-two years. Of this number, there were one hundred and two girls and fifty-five boys. A list of five needs was proposed.

1. The need to belong to a group of like-minded people, to have a large number of friends and relatives.

2. The need to be like strong, smart, fashionable, authoritative people.

3. The need to have a large amount of money and (or) movable and immovable property.

4. The need to be a leader of the highest possible rank, to have great authority.

5. The need to have a broad outlook, deep professional values, constantly developing one's creative abilities.

Students were also asked to assess the severity of their emotional stress. For this purpose, a scale ranging from zero to ten, where the value of zero corresponded to absence of stress, and the value of then indicated maximum severity. It should be noted that emotional stress is characteristic of students of both medical and non-medical branches during training (Bacchi and Licino, 2017; Bruffaerts et al., 2018; Donven et al., 2019; Ershens et al., 2019; Fawzy and Hamed, 2017). At the same time, in the context of the COVID-19 pandemic, psychological problems have become more pronounced (Caligiuri et al.; 2020; Chandratre, 2020). In addition, students were simultaneously recommended to subjectively assess the severity of their feelings of joy and happiness. The rating scale used the same range, where the value of zero stood for absence of feelings of joy and happiness, while the value of ten signified presence of these feelings in full. The aim of the study was to find out which need was accompanied by least emotional stress and the most pronounced feeling of joy and happiness. The study was conducted in February - March 2021.

\section{Results}

The list of needs presented to students for rating was not chosen randomly. In our opinion, the requirements listed represent manifestations of the basic need for security. These needs, when attempted to be satisfied, are "channels" for addressing the imbalance associated with a lack of a sense of security. The first such "channel" is the desire to belong to a group of people, in particular, like-minded people or relatives. To this we can add that it was traditionally believed, and in some countries still is, that the larger the family, the more children there are in it, the safer each family member is. Given this safety criterion, people in developing countries, who usually have more children, should not feel less safe than people in developed countries, where families are much smaller. This is not always the case, which can be explained by the fact that in developed countries the state, with the help of a system of laws, the army, and the police, assumes the function of ensuring the safety of its citizens.

The second "channel" for establishing equivalence with a lack of a sense of security can be called imitation, in one form or another, of those whom a person considers stronger. Imitation can be expressed, for instance, by training in the gym to become stronger to counter possible dangers. Some people start to practice martial arts, so that, if necessary, they can behave like movie characters. Part of the population believes that only possession of arms, and the skills to use them, can help, as seen in films and novels, to ensure personal safety. One may also imitate the manners and clothing of the inhabitants of countries that are considered strong and influential. A form of this type of imitation is application of a foreign language, even where not necessary, like use of English for names of shops, business centers, and city neighborhoods. 
The third "channel" for establishing equivalence is stockpiling. This includes accumulation of "doomsday" food and medical supplies or acquisition of ever more property. The desire for as much money as possible, even if it does not seem necessary, is also an attempt to eliminate the lack of a sense of security.

The fourth "channel" for establishing equivalence is acquisition of power. Many people think that the more power they have, the safer they are. Judging by numerous examples of power struggles at different levels, this "channel" of establishing equivalence is considered very effective for obtaining a sense of security.

And, finally, the fifth "channel" represents acquisition of a sense of security by obtaining new knowledge. A knowledgeable, prepared person can ensure their safety in various situations, and be an example to others in difficult times. Knowledgeable, competent specialists are also the ones most sought after on the labor market.

Having needs means always finding ways to meet them, in other words, the "channels" for establishing equivalence. Being successful in this search means that the individual in question has had a positive experience of satisfying their needs and wish to repeat it. Persistence of needs on the other hand may have an individual trying again and again to find their "channel" of establishing equivalence. Unsuccessful attempts can lead either to the rejection of the need and its subsequent replacement, or, if the imbalance is very pronounced, to serious consequences for human health.

The results of the student survey showed that the first need on our list was the most relevant for eleven students $(7 \%)$, the second need for nine students, the third need for seventy-one students, $(45.22 \%)$, the fourth need for thirty-four students $(21.65 \%)$, and the fifth need for thirty-two students $(20.38 \%)$. When determining the severity of emotional stress in students, it was found that the maximum severity (six to ten points on the scale) was perceived by those who considered the third need as the most significant. This condition was observed in sixtythree students out of seventy-one $(88.73 \%)$.

The least number of students to feel pronounced emotional stress (six to ten points on the scale) was found among those who prioritized the fifth need. Of the thirty-two students, only seven $(21.87 \%)$ showed signs of emotional stress.

When analyzing the intensity of feelings of joy and happiness, the most interesting results, in our opinion, were also found in students who preferred the third and fifth needs. Thus, the lowest number of students who feel joy and happiness (six to ten points on the scale) was found in those who preferred the third need. There were eleven such students (15.49\%) out of seventyone. At the same time, the greatest number of students who felt joyful and happy was found among those who identified the fifth need as the most significant. Students with these feelings numbered twenty-two out of thirty-two $(68.75 \%)$.

Thus, the largest number of students with severe emotional stress, and, at the same time, the smallest number of students who experienced feelings of joy and happiness, was observed under those who picked the third need as their top choice.

This imbalance was accompanied by the formation of such "channels" for establishing equivalence as attempts to earn more and more money or purchase new property. But, apparently, for most students, these attempts did not lead to the elimination of the lack of a sense of security. This can be indicated by their significant emotional stress and, most often, lack of the desired result.

Achievement of such result is usually indicated by positive emotions, which were rarely found in students of this group.

At the same time, if the imbalance associated with a lack of a sense of security was caused by a lack of knowledge, the situation was different. The students who picked the fifth need as 
their priority and the corresponding channel for establishing equivalence, showed the least degree of emotional stress and the greatest degree of positive emotions. In our opinion, the students of this group, despite their constant need for new knowledge, successfully eliminated the imbalance and established equivalence. The achievement of the desired result was indicated by the fact that the students of the group in question had the greatest number of positive emotions. We should add that imbalances can also occur due to formation of high-level needs in a person, or, as they were defined by Maslow (1963), meta-needs. Maslow, speaking about self-actualized people, wrote: "They [...] devote themselves to the search for [...] the values of Being - the search for the main, eternal, underlying values that cannot be subordinated to any other, more important ones. [...] These values [...] arise in the role of needs and behave accordingly [...] These are meta-needs" (Maslow, 1963). The fifth need may be, in our opinion, regarded as a meta-need. In addition, the imbalance, in our opinion, is the meaning of life as a highest example of meta-needs. The higher the level of understanding of what a person considers to be the meaning of life, the more pronounced is the imbalance, the more effort is required to eliminate it, to find "channels" for establishing equivalence. But the easier it is, according to the dominant principle (Sudakov, 1993), the more readily less significant imbalances are eliminated. That is why we believe the students who chose the fifth "channel" are in most beneficial position.

\section{Discussion}

From human behavior, on the one hand, it can be understood that the elimination of the lack of a sense of security occurs through a combination of all five "channels" allocated by us to form an equivalent response. On the other hand, it is always possible to identify the "channel" of establishing equivalence, which a person considers to be their main one. At the same time, the question arises as to what exactly determines the individual rate of establishing equivalence.

The answer to this question, in our opinion, can be given when the problem of human potential is taken into consideration. Potential is associated with an innate imbalance. As noted earlier (Avilov, 2019), from the point of view of our theory the imbalance already exists at the zygote level and is determined by the initial level of disequilibrium. The higher the level of disequilibrium, the higher, all other things being equal, level of imbalances that need to be eliminated during one's life. It is this initial level of disequilibrium that, in our opinion, represents the potential of an organism. During one's life, there is a continuous movement from disequilibrium to equilibrium, the final manifestation of which is the death of the organism or, in other words, the realization of the individual's potential. Thus, within the framework of our theory of functional equivalence, under the potential of a living system, we understand the innate capabilities of an organism and its individual systems as determined by the initial disequilibrium, to eliminate the manifestations of imbalance that arise in the course of life. This potential has to be realized in the process of individual development, the only question is how fast this happens, how quickly the body "self-actualizes" given the potential. If the severity of the imbalance is minimal, that is, the excess of information, physical and chemical manifestations of the results of functioning is removed with the necessary speed with the help of stimuli coming from the external and internal environment of the body, the reduction of the potential, in our opinion, is minimal. That is, the movement from disequilibrium to equilibrium slows down. In case of a decrease in the flow of information, physical and chemical stimuli for a certain period of time, due to the potential of the body, the functioning is maintained at the same or even increased level, including with respect to the search for the missing stimuli, but is always equivalent to the situation and/or its significance, which is determined subjectively. 
If the stimulation of the body remains reduced, or completely stops, this results in either the death of the body, as, for example, given absence of oxygen supply or a compensatory, equivalent decrease in the volume and mass of the body's tissues, as well as a change in nature of its functioning. Such a variant of establishing equivalence can be observed, for example, in patients who stay in a supine position for a long time. On the other hand, given an excessive supply of information, physical and chemical stimuli from the external and, sometimes, also the internal environment of the body, the potential is additionally spent on achieving equivalence by gradually forming an increased volume and mass of tissues, which in this case represent the main "channels" for establishing equivalence. Such changes can be observed, for example, during sports training. In addition, it is well known that drowsiness occurs when a large amount of food is consumed, a fact we regard as an emergency provision of equivalence by removal of the information component of the results of vital activity. Given either insufficient or excessive input of incentives, an increased level of spending potential can lead to disease and/or death of the body, since the movement from disequilibrium to equilibrium occurs at an accelerated pace. In general, however, the establishment of equivalence is a form of slowing down the movement to the equilibrium state rather than acting against this equilibrium. At the same time, by increasing "abilities" (by establishing equivalence through a particular "channel"), we, to a certain extent, lose "capabilities" (by reducing the level of disequilibrium, or the potential of the body and its individual organs and systems).

In our opinion, people with a high level of potential are more likely to suffer from an insufficient supply of incentives. They are therefore looking for different ways to realize their potential and are most likely what we call the "engines of progress". People with a medium or low level of potential are most likely to experience problems associated with excessive input of stimuli from the external environment. Advertising, especially in the context of globalization (Avilov, 2020), can make these people try to "be like everyone else", even at the risk to one's health. Through advertising, the need to buy new and new goods and services is imposed on individuals (Sahni and Nair, 2020). Advertising can further reinforce the imbalances associated with a lack of a person's sense of security. Because of this, the desire to be on someone's "team", the desire to accumulate something, to imitate, to possess money, power, or knowledge can be made more acute. The desire for knowledge can then be the main imbalance, or a means by which the other imbalances can be eliminated.

It should be noted that the behavior of a person can be understood whether they have pronounced imbalances or not. This behavior will be determined by reactions such as those to emotional stress (Selye, 1952). From the point of view of the theory of functional systems, emotional stress is formed in all conflict situations in which the subject cannot meet the needs available to them (Bogler and Somech, 2019; Lacuta, 2019). Nevertheless, according to the theory of functional equivalence, the view of the causes of stress may be somewhat different. The meaning of a stress reaction, in our opinion, is a response to a situation where the rate of development of an imbalance due to the impact or sudden absence of exposure to information, physical or chemical factors exceeds the rate of establishing equivalence. In this case, an attempt is made to generate an emergency equivalent response through different "channels". As a result, there is a change in the functioning of almost all body systems. However, since the body's response is urgent, stress is in this phase of its development accompanied by a significant disintegration in the functioning of its functional systems, as well as negative emotions (Sudakov, 1993). If the imbalance exists for a long time, then in order to find "channels" for establishing equivalence, chronic emotional stress develops in the body. In our opinion, its manifestations against the backdrop of negative emotions can be described as states of frustration, anxiety, and cognitive dissonance. We believe that it is the long-term lack of 
elimination of imbalances by establishing equivalence that leads to consequences such as burnout syndrome, chronic fatigue syndrome, neuroses, psychosomatic diseases, and, as a result, to a reduction in life expectancy. The needs can however also be met. Then, in case of establishment of a full equivalence, positive emotions are formed in a person and there is a transition from disorganization to consistency in the work of functional systems (Sudakov, 1993).

Thus, the development of emotional stress depends both on the potential of the body and on the number of additional imbalances created and not eliminated. The obstacles that arise in the way of trying to establish equivalence also contribute to formation of emotional stress. Thus, the COVID-19 pandemic has made it difficult to address both natural and artificial imbalances (Chandratre, 2020). At the same time, under the same conditions, the psychological comfort of the students we examined depended on what imbalance they considered the most important to eliminate. The greatest obstacles to eliminating the imbalance associated with the lack of a sense of security arose when students tried to earn more money to establish equivalence, or to own of more property. Pronounced emotional stress and a lack of positive emotions in the implementation of this "channel" of establishing equivalence can be highly dangerous to students' wellbeing. On the other hand, if students believed that the main reason for the lack of a sense of security was a lack of knowledge, the psychological consequences were different. The obstacles that arise in the pursuit of new knowledge are usually quite easy to overcome. That is why, in our opinion, the emotional stress of the students was expressed only mildly, and positive emotions, indicating that the equivalence was established and the lack of knowledge eliminated, were found most often in the same group.

It is well known that in developed countries human potential is not spent solely on correcting imbalances caused by the lack of opportunities for simple survival. A person must find additional directions for spending their potential. It is most often spent to eliminate imbalances associated with a real or advertising-imposed need to purchase goods and services. Eliminating imbalances in this way leads to development of positive emotions. The better an ad works, the more purchases occur. The more purchases, the more often positive emotions and feelings of joy arise. This is the meaning of economic activity (Avilov, 2020). Moreover, the positive emotions and optimistic behavior contribute to effectiveness of work (Bogler and Somech, 2019). These emotions are capable to push a person to continually state new purposes, to conscious or subconscious formation of imbalances, to receive positive rather than desirable emotions (Haggag et al., 2019) after establishment of equivalence. A kind of dependency may arise along with "addictive consumption", a serious problem in many countries (Reith, 2004). The third need may thus be constantly satisfied if there is no lack of potential or resources needed, but this may exceed a person's capacity to establish equivalence, especially during the COVID-19 pandemic, which brought with it pronounced emotional stress, due to disruption of the usual way of life and changes in the structure of consumption, with emotionally unstable people suffering most of all (Lacuta, 2019; Caliguiri et al.,2020). We believe that in this situation it is more beneficial to shift from the third need to the fifth need, in other words, to spend one's potential to get more services than goods, because acquiring knowledge practically always means getting more services. Very often, it is easy to find ways to get these services free of charge or having spent only little amount of money. That is why the shift mentioned could lead to less pronounced emotional stress, less health issues (Liu and Menegatti, 2019) and health-related human resource management (Huettermann and Bruch, 2019; Zagenczyk et al., 2020). In our opinion, optimizing one's needs are important for all individuals with lower amount of potential left, including those who are nearing retirement (Mazzonna and Peracchi, 2017; Menoncin and Vergally, 2021). 
Therefore, how happy and healthy we can be depending on what we consider the main imbalance for ourselves, on our chosen "channel" for establishing equivalence.

\section{Conclusion}

The theory of functional equivalence, in our opinion, can help to generalize the available data concerning human behavior, as well as to outline new research directions. The proposed view on the nature of needs can help us understand the relationship between a person's potential, emotional stress, and behavioral responses. At the same time, human behavior is largely determined by a lack of a sense of security. Eliminating this imbalance, finding a "channel" for establishing equivalence is accompanied by a decrease in emotional stress and formation of positive emotions - a person feels happier. As the Dalai Lama wrote: "Whether you belong to a particular religion or not, whether you belong to this religion or that, the main goal of our life is happiness, the main motivation of our life is the pursuit of happiness" (Dalai- Lama, 2004). As a result of our research, it turned out that students who believed their main to be lack of knowledge were happier than those students who believed it was most important for them to eliminate their lack of material resources. For preservation of psychological comfort and human health it is desirable to change one's preferences accordingly. It is quite difficult to reconstruct the hierarchy of imbalances formed through upbringing and individual training (Kelly and Barker, 2016). At the same time, there are successful examples of such changes (Avilov, 2020). Clearly, the question arises concerning the possibility of rapid formation of the need for selfactualization in order to find the "channels" for establishing equivalence such as are the most resistant to external influences. To answer it, forty students were selected from the group of sixty-three who chose the third and suffered the most acute decrease in the ability to meet these needs. For these individuals, psychotherapy sessions including coaching (Oreopoulos and Petronijevic, 2018; Marrone et al., 2021) were conducted individually during the week according to our method. Each session lasted one hour. The purpose of the sessions was to create a new imbalance to take up the most important place in their individual hierarchies of needs - the need for self-actualization (the fifth need on our list). In parallel, work was carried out to eliminate the new imbalance by finding a new "channel" for establishing equivalence. The criterion by which it was judged that such new "channel" was found was an increase in the number of positive emotions experienced by students during the day, as well as a decrease in their level of emotional stress. It turned out that after a week of psychotherapy, thirty-four students out of forty had increased joyful expectations of life, and on the scale of subjective assessment of emotional stress, none of them indicated values greater than three points (weakly expressed emotional stress). The lack of success in working with the remaining six students who were also offered psychotherapy can be explained by the fact that the period of one week was insufficient to find an appropriate "channel" for establishing equivalence for them. We also compared those students who were offered psychotherapy with those who were not offered it. It turned out that for all students who were given a course of psychotherapy, their subjectively assessed level of emotional stress decreased after a week that followed the end of the course despite the ongoing COVID-19 pandemic. In comparison, among those students who did not receive a course of psychotherapy, a decrease in the level of emotional stress (less than five points on the scale) were observed in only three out of twenty-three people after a week. It is therefore apparently possible to change an individual subjective rating of needs. If the set of needs is tailored to one's potential, the person will be happier and will be able to understand what the Dalai Lama meant when he spoke about limiting individual consumption. The Dalai Lama was right! 


\section{References}

Avilov, O. V. (2019). Formation of life meaning and health at the foundation of functional equivalence theory. The European Journal of Natural history, 3, 72-74.

Avilov, O. V. (2020). Psychology of the safe consumption at the foundation of functional equivalence theory. Economic and Social Development: Proceedings of the 50 $0^{\text {th }}$ international Conference, Chelyabinsk: Online Edition, 359-364.

Bacchi, S., \& Licino, J. (2017). Resilience and psychological distress in psychology and medical students. Acad. Psychiatry, 41, 185-188.

Bogler, R., \& Somech, A. (2019). Psychological capital, team resources and organizational citizenship behavior. The Journal of Psychology, 153(8), 784-802.

Bruffaerts, R., Mortier, P., Kiekens C. et al. (2018). Mental health problems in college freshmen: Prevalence and academic functioning. Journal of Affective Disorders, 225, 97-103.

Caligiuri, P., De Cieri, H., Minbaeva, D., Verbeke, A., \& Zimmermann, A. (2020). International HRM insights for navigating the COVID-19 pandemic: Implications for future research and practice. The Journal of International Business Studies, 51(5), 697-713.

Chandratre, S. (2020). Medical students and COVID - 19: Challenges and Supportive Strategies. Journal of Medical Education and Curricular Development, 7, 1-2.

Donven, R., Remmerswaal, M., \& Zoutenbier, R. (2019). Do altruistic mental health care providers have better treatment outcomes? The journal of human Resources, 54(2), 310-341.

Ershens, R., Keifenheim, K. E., Herrman-Werner, A. et al (2019). Proffessional burnout among medical students: Systematic literature review and meta - analysis. Medical Teacher, 41(2), 172-183.

Fawsy, M., \& Hamed, A. (2017). Prevalence of psychological stress, depression and anxiety among medical students in Egypt. Psychiatry research, 255, 186-194.

Haggag, K., Pope, D.G., Bryant-Lees, K. B., \& Bos, M. W. (2019). Attribution bias in consumer choice. The Review of Economic Studies, 86(5), 2136-2183.

His Holiness the Dalai - Lama, Cutler, H. C. (2004). The art of happiness. A Handbook for Living. Kiev, Moscow, Saint - Peterberg «Sofia» Publ.

Huettermann, H., \& Bruch, H. (2019). Mutual gains? Health - related HRM, collective well - being and organizational performance. Journal of Management Studies, 56(6), 1045-1072.

Kelly, M. P., \& Barker, M. (2016). Why is changing health-related behavior so difficult? Public Health, 136, 109116

Lacuta, P. (2019). Personality trait interactions in risk for and protection against social anxiety symptoms. The Journal of Psychology, 153(6), 599-614.

Liu, D., \& Menegatti, M. (2019). Optimal saving and health prevention. Journal of Economics, 128, 177-191.

Marrone, J. A., Quigley, N. R., Prussia, G. E., \& Dienhart, J. (2021). Can supportive coaching behaviors facilitate boundary spanning and raise job satisfaction? An indirect-effect model. Journal of Management, 24(3), 231245.

Maslow, A. H. (1963). Further notes on psychology of being. Journal of Humanistic Psychology, 3, 12-135.

Mazonna, F., \& Peracchi, F. (2017). Unhealthy retirement? The Journal of Human Resources, 52(1), 128-151.

Menoncin, F., \& Vergally, S. (2021). Optimal stopping time, consumption, labour, and portfolio decision for a pension scheme. Journal of Economics, 132, 67-98.

Oreopoulos, P., \& Petronijevic, U. (2018). Student Coaching: How Far Can Technology Go? The Journal of Human Resources, 53(2), 299-329.

Reith, G. (2004). Consumption and its discontents: Addiction, identity and the problem of freedom. The British Journal of Sociology, 55(2), 697-713.

Sahni, N. S., \& Nair, H. S. (2020). Does advertising serve as a signal? Evidence from a field experiment in mobile search. The Review of Economic Studies, 87(3), 1529-1564.

Selye, H. (1952). The story of the adaptation syndrome. Montreal (Canada). Acta inc. Medical. Publ.

Sudakov, K. V. (1993). Brain neuronal mechanisms of motivation and reinforcement: System organization of behavior. Integrative Physiology and Behavioral Sciences, 28(4), 396-407.

Zagenczyk, T. J., Powell, E. E., \& Scott, K. L. (2020). How exhausting!? Emotional crossover in organizational social networks. Journal of Management Studies, 57(8), 1589-1609. 